\title{
THE
}

\section{Quantum Effects in the Dynamics of the One-Dimensional Planar Antiferromagnet}

Gerhard Müller

University of Rhode Island, gmuller@uri.edu

Harry Thomas

Marcos W. Puga

Hans Beck

Follow this and additional works at: https://digitalcommons.uri.edu/phys_facpubs

Terms of Use

All rights reserved under copyright.

\section{Citation/Publisher Attribution}

Gerhard Müller, Harry Thomas, Marcos W. Puga and Hans Beck. Quantum effects in the dynamics of the one-dimensional planar antiferromagnet, in Physics in one dimension, J. Bernasconi and T. Schneider (Eds.), Springer Series in Solid State Sciences (Springer, Berlin-Heidelberg-New York, 1981), pp. 165-168. Available at: http://dx.doi.org/10.1007/978-3-642-81592-8_17 


\title{
Quantum Effects in the Dynamics of the One-Dimensional Planar Antiferromagnet
}

\author{
Gerhard Müller, 1 Harry Thomas, 10 Marcos W. Puga, 느 and Hans Beck는 \\ ${ }^{1}$ Institut für Physik, Universität Basel, CH-4056 Basel, Switzerland \\ 2 Institut de Physique de l'Université, $\mathrm{CH}-2000$ Neuchâtel, Switzerland
}

In a recent publication [1] we found that the $T=0$ dynamics of the $1 \mathrm{D} s=\frac{1}{2}$ Heisenberg antiferromagnet (HB AF) with nearest-neighbor exchange interaction $J$ is almost completely dominated by a particular continuum of excitations (called spin-wave continuum, SWC) bounded by the dispersion branches $\epsilon_{1}(q)=(\pi J / 2) \sin q$ and $\epsilon_{2}(q)=\pi J \sin (q / 2)$. It differs markedly from the classical ID HB AF where the spectral weight is concentrated on a single branch of spin-waves. The result for the structure function $S_{\mu \mu}(q, \omega) \equiv\left\langle S_{l}^{\mu} S_{l^{\prime}}^{\mu}\right\rangle_{q, \omega}$, which is a special case of Eq. (6), is in good agreement with low-Tneutron scattering data on CPC [2] concerning excitation energies, lineshapes and integrated intensity.

In this paper we outline an extension to the planar $s=\frac{1}{2} \mathrm{AF}$ with Hamiltonian

$$
H=\sum_{l=1}^{N}\left\{J_{\perp}\left(S_{l}^{x} S_{l+1}^{x}+S_{l}^{y} S_{l+1}^{y}\right)+J_{z} S_{l}^{z} S_{l+1}^{z}\right\}, \quad J_{\perp} \equiv J>0, \quad 0<J_{z}<J .
$$

By using finite-chain calculations on systems with 4 to 10 spins, we again find a special class of excitations forming a two-parameter continuum (SWC) in $(q, \omega)$-space which dominates $S_{\mu \mu}(q, \omega)$ at $T=0$. Further, we can identify each one of these excitations with a member of the 'class C' of non-bound states defined in the framework of Bethe's formalism. By use of des Cloizeaux and Gaudin's method [3], we obtain the SWC energies

$$
\omega_{m}(q)=\frac{\pi J \sin \theta}{\theta} \sin \frac{q}{2} \cos \left(\frac{q}{2}-\frac{q_{m}}{2}\right) ; \quad q \geq 0, \quad 0 \leq q_{m} \leq q,
$$

where $\theta=\arccos \left(J_{z} / J_{\perp}\right)$, and $q_{m}$ labels the different dispersion branches of the SWC. In particular, $q_{m}=0$ and $q_{m}=q$ yield the lower and the upper boundary, respectively,

$$
\epsilon_{1}(q)=\frac{\pi J \sin \theta}{2 \theta} \sin q, \quad \epsilon_{2}(q)=\frac{\pi J \sin \theta}{\theta} \sin \frac{q}{2} .
$$

From (2) we deduce the density of states in the SWC

$$
D(q, \omega)=\frac{N}{2 \pi}\left\{\epsilon_{2}^{2}(q)-\omega^{2}\right\}^{-1 / 2} ; \quad \epsilon_{1}(q) \leq \omega \leq \epsilon_{2}(q) .
$$

In analogy to our result for the $\mathrm{HB} \operatorname{AF}\left(J_{z}=J_{\perp}\right)$ and to exact results for the $\mathrm{XY} \operatorname{AF}\left(J_{z}=0\right)$ [1], we conjecture that $S_{z z}(q, \omega)$ can be written as a product $M(q, \omega) \cdot D(q, \omega)$ with

$$
M(q, \omega)=\text { const } \cdot\left\{\frac{\epsilon_{2}^{2}(q)-\omega^{2}}{\omega^{2}-\epsilon_{1}^{2}(q)}\right\}^{\alpha}
$$

representing the squared matrix elements $\left|\left\langle\mathrm{G}\left|S^{z}(q)\right| \mathrm{SWC}\right\rangle\right|^{2}$ between the non-degenerate ground state and the SWC excitations. The exponent $\alpha$ can be related to exponents of the Baxter model 
[4] yielding $\alpha(\theta)=(\pi / 2-\theta) /(\pi-\theta)$. Thus we are led to the analytic expression

$$
S_{z z}(q, \omega)=\frac{2 A}{B(1-\alpha, 1 / 2+\alpha)} \frac{\Theta\left(\omega-\epsilon_{1}(q)\right) \Theta\left(\epsilon_{2}(q)-\omega\right)}{\left(\omega^{2}-\epsilon_{1}^{2}(q)\right)^{\alpha}\left(\epsilon_{2}^{2}(q)-\omega^{2}\right)^{1 / 2-\alpha}},
$$

where the Beta function serves as a convenient normalizing factor, and the constant $A$ will be determined below. In the limit $J_{z} / J_{\perp}=0(\theta=\pi / 2)$, this result is exact [1].

Fig. 1 shows $S_{z z}(q, \omega)$ at fixed $q=4 \pi / 5$ as a function of $\omega$ for various anisotropies. In the $X Y$ limit $\alpha=0 M(q, \omega)$ is a constant and $S_{z z}(q, \omega)$ reflects the SWC density of states (5) with a squareroot divergence at $\epsilon_{2}(q)$ and a finite step at $\epsilon_{1}(q)$. For intermediate anisotropies $(0<\alpha<1 / 2)$ $M(q, \omega)$ is no longer a constant, but diverges at $\epsilon_{1}(q)$ and vanishes at $\epsilon_{2}(q)$. This leads to a weaker singularity in $S_{z z}(q, \omega)$ at $\epsilon_{2}(q)$ and gives rise to a second divergence at $\epsilon_{2}(q)$. We therefore expect that inelastic neutron-scattering experiments will reveal a two-peak structure at low $T$. In the HB AF limit $\alpha=1 / 2)$ the matrix elements (5) vanish sufficiently rapidly at $\epsilon_{2}(q)$ in order to overcome the density-of-states singularity. Most of the spectral weight is now concentrated at $\epsilon_{1}(q)$. It should be remarked that the spectrum contributing to $S_{z z}(q, \omega)$ has no gap at $q=0$ or $q=\pi$ in the whole range $0 \leq J_{z} / J_{\perp} \leq 1$. This is a clear quantum effect, for it is in marked contrast to classical spin-wave theory. Here, the excitations contributing to $S_{z z}(q, \omega)$ consist of a single branch (in the extended zone) [5]

$$
\epsilon_{\mathrm{cl}}(q)=2 J s\left\{(1-\cos q)\left[1+\left(J_{z} / J\right) \cos q\right]\right\}^{1 / 2},
$$

which has a gap at $q=\pi$ increasing with planar anisotropy. Note that in the quantum case the spectral weight of $S_{z z}(\pi, \omega)$ is also redistributed, with increasing anisotropy, towards higher energies, but rather in terms of a varying exponent than in terms of an energy gap.

An important check for the validity of our result (6) is the application of the sum rule [6]

$$
K_{z z}(q) \equiv \int_{0}^{\infty} \frac{\mathrm{d} \omega}{2 \pi} \omega S_{z z}(q, \omega)=-2 J F_{x}(1-\cos q),
$$

where the nearest-neighbor correlation function $F_{x} \equiv\left\langle S_{l}^{x} S_{l+1}^{x}\right\rangle$ has been calculated exactly by Yang and Yang [7]. The first frequency moment of (6)

$$
K_{z z}(q)=\frac{A J \sin \theta}{4 \theta}(1-\cos q)
$$

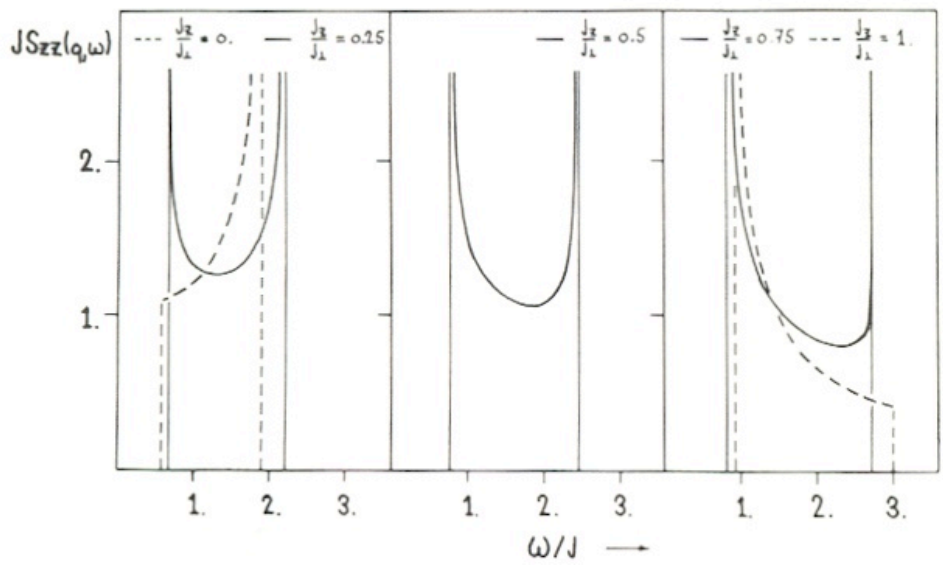

Figure 1. Out-of-plane structure function $S_{z z}(q, \omega)$ of Eq. (6) versus $\omega$ at fixed $q=4 \pi / 5$ for various anisotropies $J_{z} / J_{\perp}$. The prefactor $A$ has been determined for this figure via the sum rule. 
reproduces the exact $q$-dependence irrespective of the anisotropy. The integrated intensity is obtained as

$$
\begin{gathered}
I_{z z}(q) \equiv \int_{0}^{\infty} \frac{\mathrm{d} \omega}{2 \pi} S_{z z}(q, \omega)=\frac{A}{2 \pi} \tan \frac{q}{2}{ }_{2} F_{1}\left(\frac{1}{2}, 1-\alpha, \frac{3}{2},-\tan ^{2} \frac{q}{2}\right), \\
I_{z z}(q) \simeq \frac{A}{4 \pi} q, \quad(q \ll 1) ; \quad I_{z z}(\pi)=\frac{A}{4 \sqrt{\pi}} \frac{\Gamma(1 / 2-\alpha)}{\Gamma(1-\alpha)} .
\end{gathered}
$$

In the $X Y$ limit the linear term is already the exact result for all $q$. In the $\mathrm{HB} \operatorname{AF}$ limit $I_{z z}(q)$ has a logarithmic divergence at $q=\pi$. The asymptotic behaviour of the correlations in real space is $\left\langle S_{l}^{z} S_{l+R}^{z}\right\rangle \sim(-1)^{R} / R^{2(1-\alpha)}$. Thus, the out-of-plane correlations are strongest in the isotropic limit. With increasing anisotropy the spins become more and more correlated in the easy plane, and hence the fluctuations out of the plane are increasingly suppressed. Another important static quantity is the $q$-dependent susceptibility

$$
\begin{gathered}
\chi_{z z}(q) \equiv \int_{0}^{\infty} \frac{\mathrm{d} \omega}{2 \pi} \omega^{-1} S_{z z}(q, \omega)=\frac{A \theta}{\pi^{2} J \sin \theta}{ }_{2} F_{1}\left(1, \alpha+\frac{1}{2}, \frac{3}{2}, \sin ^{2} \frac{q}{2}\right), \\
\chi_{z z}(0)=\frac{A \theta}{\pi^{2} J \sin \theta}, \quad \chi_{z z}(q) \sim(\pi-q)^{-2 \alpha} \quad(q \rightarrow \pi) .
\end{gathered}
$$

The exact result for the uniform susceptibility is already available [7]: $\chi_{z z}=\theta /[J \pi(\pi-\theta) \sin \theta]$. Hence, with the sum rule result (8), (9) and with the uniform susceptibility (13), we have two independent ways of determining $A$, yielding

$$
A_{K}(\theta)=-8 \theta F_{x}(\theta) / \sin \theta, \quad A_{\chi}(\theta)=\pi /(\pi-\theta) .
$$

The difference between the two values is not greater than a few percent, except close to the HB AF limit, where it reaches $15 \%$. This discrepancy in the isotropic limit is understood and extensively discussed in Ref. [1].

We conclude by noting that the present approach also works for the in-plane component of the structure function $S_{x x}(q, \omega)$. In this case there are two dominating spin-wave continua, one of which is again confined between the branches $\epsilon_{1}(q)$ and $\epsilon_{2}(q)$ of Eq. (3) and the second one between the boundaries $\epsilon_{1}(\pi-q)=\epsilon_{1}(q)$ and $\epsilon_{2}(\pi-q)$. We find that the SWC contributions to $S_{x x}(q, \omega)$ can be written as a sum of two terms of the form (6) with the boundaries in one of them replaced accordingly. The prefactors and the exponents in the two terms are different functions of the planar anisotropy. Hence the corresponding lineshapes are predicted to be even more complex than those of $S_{z z}(q, \omega)$. Furthermore we can show that an ansatz of the form (6) also holds for the isotropic $s>\frac{1}{2} \mathrm{HB}$ AF with an exponent $\alpha$ which in this case depends on the spin quantum number $s$. Here we find that with increasing $s$ the lineshape of $S_{\mu \mu}(q, \omega)$ becomes more symmetric. In the limit $s \rightarrow \infty$ the exact classical result is recovered. More details will be published elsewhere.

\section{Acknowledgments}

This work is supported by the Swiss National Science Foundation. We have used a modified cmpj.sty style file.

\section{References}

1. G. Müller, H. Thomas, H. Beck and J.C. Bonner, submitted to Phys.Rev. B.

2. I.V. Heilmann, G. Shirane, Y. Endoh, R.J. Birgenau, and S.L. Holt, Phys. Rev. B 18, 3530 (1978).

3. J. des Cloizeaux and M. Gaudin, J.Math.Phys. 7, 1384 (1966).

4. A. Luther and I. Peschel, Phys.Rev. B 12, 3908 (1975).

5. R.E. Dietz, L.R. Walker, F.S.L. Hsu, W.H. Haemmerle, B. Vis, C.K. Chau, and H. Weinstock, Sol.State Comm. 12, 1185 (1974).

6. P.C. Hohenberg and W.F. Brinkman, Phys.Rev. B 10, 128 (1974).

7. C.N. Yang and C.P. Yang, Phys.Rev. 151, 258 (1966). 